\title{
Aprendizaje de competencias científicas versus aprendizaje de contenidos específicos. Una propuesta de evaluación
}

\section{Learning of scientific competencies vs learning of scientific content. An assessment proposal}

\author{
Alicia Benarroch \\ Universidad de Granada \\ aliciabb@ugr.es \\ Graciela Núńez \\ Universidad Nacional de San Juan. Argentina \\ gnunez@ffha.unsj.edu.ar
}

RESUMEN • En este trabajo se realiza una propuesta para el estudio del aprendizaje experimentado por estudiantes de 12 y 13 ańos tras la aplicación de una secuencia de enseñanza sobre el modelo corpuscular de la materia (MCM). En el marco teórico se plantea la distinción entre aprendizaje de competencias y de contenidos específicos, y se asocia el primero al cambio de nivel de esquemas explicativos puestos en juego por el estudiante, mientras que el segundo se asocia al cambio de los rendimientos totales. El tratamiento estadístico de los datos se realizó con un método multivariable usando el paquete SPSS 20.0. Los resultados muestran que el aprendizaje de competencias científicas conlleva una dificultad bastante mayor que el de contenidos específicos.

PALABRAS CLAVE: competencia científica; contenido científico; modelo corpuscular de la materia; nivel explicativo; aprendizaje.

ABSTRACT - This paper presents a proposal for studying the learning gained by 12-13 years old students after the implementation of a teaching proposal of the Matter Corpuscular Model (MCM). The theoretical framework poses the distinction between the learning of scientific competencies and the learning of scientific content and we associate the former with a change in the student's explanatory scheme levels, while the latter is associated with a change in the overall achievement. The statistical analysis of the data was performed using the 20.0 SPSS multivariate method. The results suggest that the learning of scientific competencies results more difficult than that of scientific content.

KEYWORDS: scientific competencies; scientific content; matter corpuscular model; explanatory level; learning. 


\section{INTRODUCCIÓN}

Las nuevas tendencias curriculares del siglo XXI se centran en la formación y evaluación de competencias, ya sean estas generales cognitivas, emocionales, sociales, instrumentales, etc. (Tobón, 2006). Se trata de una meta ambiciosa con la que se trata de responder a las actuales demandas socioeconómicas, ante las que se requiere sobre todo capacidad de decisión, autonomía, flexibilidad ante demandas cambiantes, trabajo en equipo, etc., del mismo modo que en los tiempos de la Revolución industrial se requerían pericias y conocimientos para el manejo de las máquinas.

Para los objetivos de este trabajo, nos centramos en el aprendizaje de competencias científicas y en una posible metodología que pueda servir para su evaluación, discriminándolo del aprendizaje de contenidos específicos, ambos en relación con el modelo corpuscular de la materia (MCM). Se trata de un contenido básico en los diseños curriculares de los cursos superiores de la enseñanza obligatoria de casi todos los países, lo que se justifica por distintos motivos, entre los que destacan (Benarroch, 2000a) su importancia para la ciencia actual, su utilidad para explicar y predecir cuestiones de la vida cotidiana, su potencialidad para favorecer el trabajo con modelos físicos, su capacidad para clarificar conceptos macroscópicos y su carácter básico en la iniciación en el ámbito de la química, entre otros. Asimismo, en el Programa para la Evaluación Internacional de los Alumnos (PISA), una competencia medida fue la de «explicar fenómenos científicamente relacionados con la estructura de la materia (modelo de partículas)» (OCDE, 2010).

Sin embargo, para que este contenido pueda serle útil al aprendiz en sus futuras actuaciones como ciudadano o en ámbitos profesionales y pueda ser aplicado en contextos prácticos, se requiere que su aprendizaje trasvase el ámbito puramente académico y que el estudiante llegue a ser poseedor de la competencia científica correspondiente que le permita resolver problemas de su vida cotidiana y actuar de modo competente ante las demandas externas.

En consecuencia, en este trabajo, partiendo de una determinada concepción de competencia científica, evidentemente diferente del propio concepto de conocimiento científico, se pretende la evaluación del aprendizaje de ambos aspectos, competencia y contenido, tras la aplicación de una intervención didáctica específica. Esta se realizó con un grupo de estudiantes de 12 y 13 años de la provincia de San Juan (Argentina).

\section{MARCO TEÓRICO}

Bajo este epígrafe se van a distinguir tres partes. En la primera, esbozaremos el concepto de competencia y los observables que podrían acompañar a su evaluación. En la segunda, el concepto de esquema explicativo, usado en algunas investigaciones previas acerca del conocimiento del estudiante. El objetivo de la tercera parte es ligar las dos anteriores mostrando la posible relación entre ambos conceptos y las ventajas que ello nos proporciona para la evaluación de competencias científicas.

\section{El concepto de competencia científica y sus cualidades destacables para este trabajo}

El concepto de competencia científica parece ser una seña de identidad de los nuevos currículos planteados en todos los niveles educativos del siglo XXI. Más allá de sus elementos diferenciadores, se podría entroncar con tendencias curriculares que poseen bastante tradición en la educación de ciencias, ya que, como señalan de Pro y Rodríguez (2010), la «ola competencial» no puede hacer tabla rasa de todo lo que se ha realizado sin ella. Por el contrario, desde la investigación en la enseñanza de las ciencias se lleva bastante tiempo reaccionando ante la enseñanza habitual de contenidos específicos de ciencias que tan cortos resultados prácticos y funcionales ofrece. El uso del término competencia podría ser consecuencia de la necesidad de superar una enseñanza que, en la mayoría de los casos, se ha redu- 
cido al aprendizaje memorístico de contenidos, hecho que conlleva la dificultad para que estos puedan ser aplicados en la vida real (Zabala y Arnau, 2007).

Más allá de su carácter polisémico, al definir competencia es usual aludir a la acción exitosa, adecuada o efectiva de una persona para desarrollar una actividad ante situaciones diversas en un ámbito concreto. Si el ámbito es el propio de las ciencias, entonces se habla de "competencia científica». Aunque las diferencias son palpables cuando se quiere precisar cómo se evalúa la «acción exitosa, adecuada o efectiva", para lo que se pretende en este trabajo, sobre la noción de competencia, interesa destacar que una competencia es una capacidad o habilidad que el estudiante pone de manifiesto durante un tiempo razonable. Esta cualidad se puede precisar con tres observables:

- Repetición: ante situaciones semejantes la reconocida competencia del sujeto se ejecuta con resultados parecidos una y otra vez (variación intracontextual).

- Generalización: ante la diversidad de variaciones del contexto en el escenario donde se ubica la competencia, el sujeto que la posee sabe responder con resultado también exitoso (variación intercontextual).

- Diferenciación: se refiere a la adecuación o acomodación de la competencia ante situaciones o circunstancias novedosas que de pronto aparecen en el escenario práctico donde se da el actuar competente del sujeto.

De este modo, como se intentará mostrar a continuación, la adquisición de una competencia podría emparentarse con la del concepto de esquema explicativo. A ello nos referiremos en el apartado siguiente.

\section{El concepto de «esquema explicativo» construido en investigaciones previas sobre el cono- cimiento de los estudiantes}

El enorme esfuerzo realizado por la investigación en didáctica de las ciencias experimentales sobre las concepciones de los estudiantes ha producido un vasto cuerpo de conocimientos en muy diferentes tópicos de ciencias, que sin duda ha contribuido a mejorar la forma en que se los enseña. En el análisis de dichas concepciones de los estudiantes, varios autores han observado una serie de regularidades entre ellas, tales como ser compartidas por sujetos de distintas culturas y edades, estar dominadas por lo perceptivo, estar marcadas por un razonamiento causal lineal tanto espacial como temporal, etc. (para una revisión más extensa, véase Marín, Benarroch y Jiménez Gómez, 2000). Sin embargo, se detectan contradicciones entre los autores con respecto a la coherencia de las concepciones. Para algunos son coherentes, mientras que para otros son difusas, poco diferenciadas y fragmentadas.

Para dar luz a este resultado contradictorio, Jiménez Gómez, Benarroch y Marín (2006) postulan que podría ocurrir que algunas respuestas de los estudiantes procedan principalmente de esquemas bien estructurados en sus sistemas de conocimiento, mientras que otras podrían proceder esencialmente de información acumulada memorísticamente, lo que daría lugar a respuestas académicas y de compromiso o de azar. Las primeras mostrarían coherencia ante el cambio de las demandas de la tarea, mientras que las segundas adolecerían de esta coherencia y manifestarían falta de regularidad y generalización ante un cambio en las demandas de las tareas.

Para los objetivos de este trabajo, las primeras respuestas son consideradas producto de los esquemas cognoscitivos de los estudiantes, mientras que las segundas podrían ser consecuencia de la mera información memorística acumulada. Los esquemas cognoscitivos forman parte de la red cognitiva inobservable del sujeto, pero tienen su correlato más cercano en el plano observable en los llamados esquemas explicativos, concebidos como regularidades en las respuestas de los estudiantes, cuando se dan las siguientes circunstancias: 
1. Repetición o extensión con que las respuestas de los estudiantes permanecen inalterables a pesar de las modificaciones de las situaciones físicas introducidas tras la aplicación de la estrategia de confrontación.

2. Generalización o extensión en la que se observan respuestas análogas ante las distintas situaciones físicas obtenidas tras la aplicación de las estrategias de variación contextual.

3. Adaptación o diferenciación de las respuestas a los factores que intervienen en la tarea (como producto de la aplicación de ambas estrategias: la de variación contextual y la de confrontación).

Este modelo cognoscitivo acerca del conocimiento del estudiante, en el que se distinguen dos planos, el observable (en el que se sitúan las respuestas de los estudiantes de las que se pueden inferir los esquemas explicativos) y el no observable (con los esquemas cognoscitivos) (Marín, 1994), ha sido utilizado satisfactoriamente durante todos estos ańos para describir, comprender y explicar las respuestas de los estudiantes ante tareas relacionadas con distintos tópicos de ciencias (naturaleza corpuscular, enlace químico, equilibrio físico, alimentación, etc.).

En la figura 1, se ha tratado de sintetizar los conceptos anteriores para facilitar la comprensión del lector.

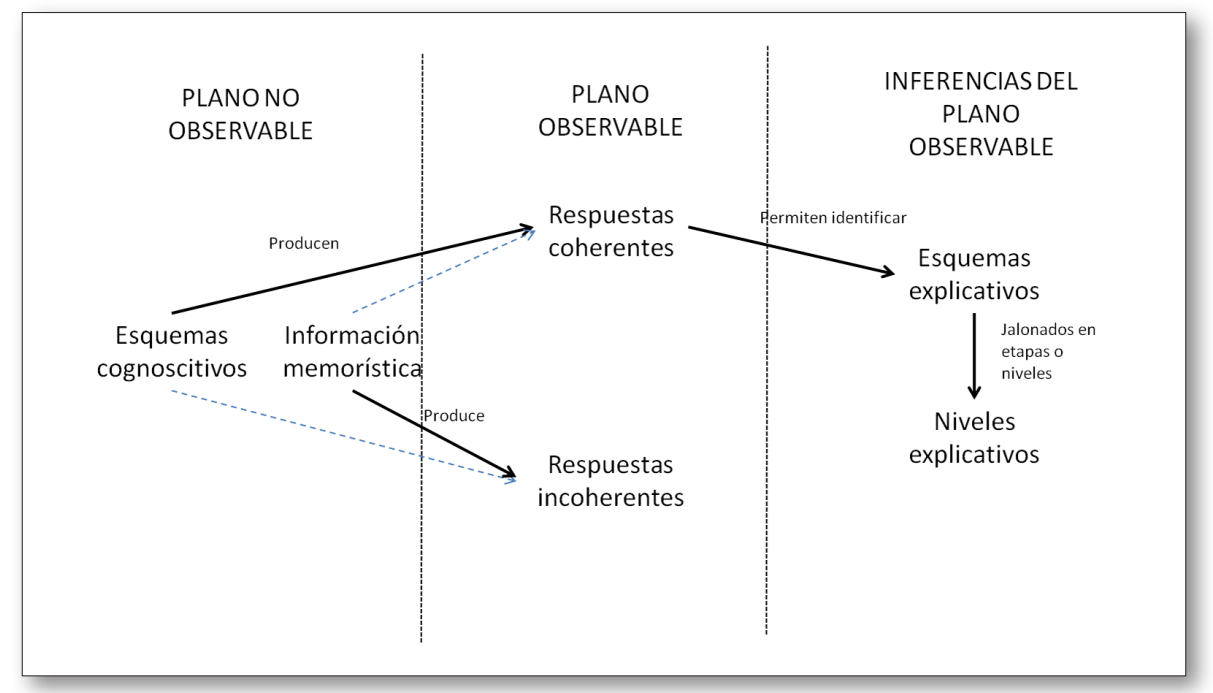

Fig. 1. Mapa de conceptos implicados en este apartado.

Los esquemas explicativos pueden ser identificados como las representaciones declarativas y externas que mejor se aproximan a los esquemas cognoscitivos. Como estos últimos, no son cuestión de todo o nada, sino que se adquieren tras un largo y dilatado proceso que acompańa al desarrollo evolutivo del estudiante y al aprendizaje acerca de ese contenido específico. Por ello, la mejor manera de describir un esquema explicativo es jerarquizándolo en etapas o niveles ordenados progresivamente, a los que hemos llamado niveles explicativos.

Para identificar los niveles explicativos, se exige del investigador la utilización de una metodología rigurosa. Muy sintéticamente esta metodología consiste en:

1. Selección de una muestra longitudinal de estudiantes con un rango de edades lo más amplio posible, dentro de lo razonable, según la dificultad inicialmente prevista para el aprendizaje del contenido de estudio. 
2. Diseño de un cuestionario capaz de activar lo máximo posible los esquemas cognoscitivos de los estudiantes. Para ello, se suele utilizar la doble estrategia de la confrontación y de la variación contextual.

3. Realización de las entrevistas individuales semiestructuradas a partir del cuestionario referido en el apartado anterior.

4. Transcripción de las entrevistas.

5. Categorización de las respuestas de los alumnos y posterior jerarquización (categorías empíricas).

6. Análisis estadístico multivariable (especialmente el análisis clúster, factorial y análisis de correspondencias) y recategorización de respuestas (categorías estructurales).

7. Delimitación de los niveles explicativos a merced del contenido evolutivo común en las explicaciones de los sujetos ante las distintas situaciones físicas planteadas.

Aplicando dicha estrategia metodológica, en Benarroch (2000b) se alcanzó la identificación de cinco niveles explicativos que jalonan el conocimiento del estudiante en el área del MCM, y que pueden ser interpretados como las formas sucesivamente más sofisticadas de razonar que presentan los estudiantes a lo largo de su estancia escolar. Estos niveles son los siguientes:

- Nivel I: conciben una imagen de materia continua y estática, basada en lo concreto y observable.

- Nivel II: mantienen la idea de continuidad de la materia pero enriquecida por elementos percibidos (burbujas, huecos, pompitas, etc.) para dar explicación a los datos empíricos. El modelo puede modificarse de una sustancia a otra para explicar los cambios de la materia.

- Nivel III: comienzan las concepciones corpusculares al considerar la materia formada por partículas invisibles, entre las que hay huecos vacíos o llenos de algo. Las explicaciones de los cambios de estado se pueden fundamentar en:

- las partículas, a las que atribuyen las propiedades percibidas (separación, acercamiento, aumento o disminución de tamaño, etc.) o en

- los huecos entre las partículas, que son utilizados para explicar los cambios macroscópicos proponiendo fondos de partículas más o menos compactos.

- Nivel IV: conciben, además de la existencia de partículas en la materia, la existencia de vacío necesario entre ellas.

- Nivel V: conciben la materia como un sistema de interacción entre partículas, moviéndose continuamente, rodeadas de vacío.

Los dos primeros niveles no requieren la utilización específica del modelo corpuscular, pues se utilizan términos macroscópicos vinculados con la experiencia directa de los fenómenos físicos. Sin embargo, en el tercer nivel se adquiere la idea de discontinuidad fundamentada en partículas materiales y fondo diferenciado. En el cuarto nivel, surge la necesidad del vacío en dicho fondo y en el quinto, se incorpora la noción de "movimiento e interacción» al subesquema anterior de "partículas y vacío». Por tanto, los tres últimos niveles sí hacen uso del modelo corpuscular, aunque en algunos casos de modo muy rudimentario.

\section{La relación entre competencia científica y esquema explicativo}

En los dos apartados anteriores de este marco teórico, se han utilizado los mismos criterios para identificar las competencias científicas y los esquemas explicativos. Estos criterios son: repetición, generalización y diferenciación. Este hecho nos hace postular una posible identidad asociativa entre ambos conceptos, pues al fin y al cabo, aun perteneciendo a líneas de investigación distintas, se refieren a la actuación observable, estable y duradera del estudiante ante las demandas externas que deben quedar 
perfectamente delimitadas de antemano. Y dicha actuación solo puede provenir de verdaderos esquemas de conocimiento.

Esta identificación tiene la ventaja de que nos permite aprovechar la investigación acumulada hasta el momento desde la didáctica de las ciencias experimentales (DCE) sobre el conocimiento del estudiante en el nuevo concepto de aprendizaje de competencias. Ya dijimos al principio del trabajo que una de las primeras tareas pendientes es encajar el tópico de la competencia en el cuerpo de conocimientos existentes en la DCE. Por otro lado, vinculado a los objetivos de este trabajo, si se admite la asociación entre ambos conceptos, podríamos evaluar el aprendizaje de la competencia científica (ligado a la mejora en la utilización del modelo cinético-corpuscular tras una intervención didáctica sobre dicha temática) mediante el cambio de los niveles explicativos alcanzados antes y después de esta.

En definitiva, en el marco teórico utilizado en la presente investigación, postulamos que:

a) El nuevo tópico de competencias científicas podría asociarse al concepto de esquema explicativo, anteriormente utilizado en investigaciones sobre el conocimiento de los estudiantes.

b) Admitido lo anterior, la evaluación del aprendizaje de competencias científicas se realizará a partir del cambio pos-pre en los niveles explicativos alcanzados por los estudiantes después y antes de la intervención educativa. Esto equivale a decir que aprender competencias implica movilizar niveles explicativos.

c) La evaluación del aprendizaje de contenidos específicos podría ser realizada a partir del rendimiento total obtenido en el cuestionario aplicado, sin discriminar entre las respuestas que proceden de los esquemas explicativos y las que no.

\section{METODOLOGÍA}

En el presente trabajo, la muestra está conformada por un grupo $(\mathrm{N}=31)$ de estudiantes de 12 y 13 años de edad de la provincia de San Juan (Argentina), que fue sometido a un proceso de enseñanza de 30 horas de duración sobre la naturaleza corpuscular de la materia por parte de una de las autoras del trabajo.

Antes y después de la intervención didáctica, se administró el cuestionario de Benarroch (1998) para delimitar: i) los niveles explicativos-competenciales (NC), y ii) los rendimientos totales (RT) de cada uno de los estudiantes.

Cabe destacar que en esta investigación el cuestionario fue administrado en gran grupo, con la profesora-investigadora realizando las experiencias contempladas en las sucesivas tareas del cuestionario, dirigiendo sus preguntas y asegurándose de que todos los estudiantes las comprendieran antes de pasar a las siguientes. Los estudiantes fueron dotados de un cuadernillo en el que iban anotando las respuestas verbales e icónicas solicitadas. Estos cuadernos de trabajo de los estudiantes constituyen las fuentes de datos que, tras el análisis cualitativo-cuantitativo, permitieron la identificación de los NC y RT previos y posteriores a la intervención didáctica.

\section{Descripción de la intervención didáctica}

Para evaluar el aprendizaje de las competencias científicas y compararlo con el de contenidos específicos, se utilizó como objeto de estudio la naturaleza corpuscular de la materia. El principal motivo por el que se realizó esta elección se basa en la amplia investigación previa realizada sobre este contenido por Benarroch (1998), lo que permite utilizar toda la potencialidad del cuestionario aplicado y disponer del espectro de respuestas obtenidas por los estudiantes de un amplio rango de edades. 
El objetivo que subyace en el conjunto de actividades de la intervención didáctica es que los estudiantes modifiquen sus modelos alternativos de la estructura de la materia y se aproximen al modelo aceptado académicamente. La intervención didáctica es una secuencia de actividades estructurada en tres tareas:

- Tarea 1: propiedades macroscópicas de sólidos, líquidos y gases. Con ella, se pretende eliminar los obstáculos macroscópicos asociados con la materia en sus tres estados (la posible inmaterialidad de los gases, la asociación prototípica gas=aire, las dificultades con los conceptos de forma, masa y volumen de los tres estados, etc.).

- Tarea 2: modelo cinético corpuscular para gases. Siguiendo criterios de la investigación didáctica, se utilizan actividades de compresión y difusión de gases coloreados para favorecer el conflicto cognitivo y conseguir que los estudiantes comiencen a pensar en la existencia de partículas moviéndose a gran velocidad en un enorme espacio vacío.

- Tarea 3: modelo cinético corpuscular para líquidos y sólidos. Aquí se busca ampliar la validez del modelo construido para los gases en la tarea anterior a sólidos y líquidos, introduciendo las modificaciones necesarias a partir de sus semejanzas y diferencias. Se comienza con los líquidos y posteriormente se amplía para los sólidos. Para ello, se realizan experiencias de mezclas, compresibilidad y dilatación. Para evidenciar que el aspecto continuo de un sistema no tiene por qué responder a su estructura interna, se realizó una actividad extra utilizando como analogía la observación, a diferentes distancias, de un vaso de precipitado que contenía bolitas de plástico.

En cada actividad, el estudiante responde por escrito individualmente a la pregunta formulada, después discute con sus compañeros en pequeño grupo y, finalmente, se le pide que manifieste nuevamente su respuesta individual. Periódicamente, se hacen puestas en común en gran grupo. La inclusión de estas estrategias pretende potenciar al máximo las prácticas metacognitivas y favorecer que el estudiante identifique su propia versión acerca de la naturaleza de su conocimiento y sobre el proceso de aprendizaje.

\section{Descripción del cuestionario usado para evaluar aprendizajes}

El cuestionario aplicado antes y después de la intervención didáctica estuvo conformado por tres tareas diseñadas por Benarroch (1998) para la delimitación de los niveles explicativos descritos en el apartado «El concepto de esquema explicativo». Las tres tareas son:

- Tarea 1: disolución de un sólido granular (acuarela amarilla) en agua.

- Tarea 2: mezcla de dos líquidos (alcohol y agua) con reducción visible de la altura total.

- Tarea 3: compresión del aire y del agua en una jeringa.

Hay que hacer notar que la selección de estas tareas no es azarosa, sino que está fundamentada en la aplicación de una de las estrategias básicas para la construcción del cuestionario, a saber, la variación contextual de las variables más relevantes del estudio. En el caso que nos ocupa, la variable contextual más relevante del estudio es el estado físico de la materia. Desde los primeros estudios sobre «atomismo» realizados por Piaget (Piaget e Inhelder, 1982) para analizar el desarrollo de nociones atomísticas y demostrar que son paralelas al desarrollo de las nociones de conservación, se sabe que la «atribución» de composiciones aditivas es precoz si el material en transformación tiene una característica granular (tarea 1); más tardía si su aspecto es continuo (tarea 2), y más aún si el material en cuestión no es perceptible (tarea 3). 
Dentro de cada tarea, se ponen en juego las otras estrategias básicas para la construcción del cuestionario: la estrategia de confrontación y la estrategia de variación contextual de variables irrelevantes del estudio.

La estrategia de confrontación implica el planteamiento de contrapruebas en cada una de las tareas. Según la teoría general de la equilibración estudiada por Piaget, las perturbaciones o contrapruebas provocan en el sujeto respuestas adaptativas o no adaptativas. Las primeras conducen a tres tipos de conductas que se manifiestan (i) mediante la rigidez del esquema asimilador (alfa), (ii) adaptación del esquema asimilador (beta) y (iii) que la perturbación no sea tal, por anticipación del esquema asimilador a las transformaciones del sistema (gamma). Se ha considerado que las respuestas posteriores a la contraprueba dan una información más cercana y significativa de la cognición del sujeto que las anteriores, debido a que dan un panorama más procesual y dinámico, pues evalúan el grado de madurez de sus esquemas de conocimiento. Las contrapruebas introducidas fueron:

- Tarea 1: agregado de pequeña cantidad de soluto coloreado al agua produciendo una solución aparentemente incolora.

- Tarea 2: comprobación empírica de la conservación del peso tras producir la disminución de volumen en la solución de agua y alcohol.

- Tarea 3: comprobación empírica de la gran compresibilidad del aire frente a la del agua en una jeringa.

Por último, se aplicó la estrategia de variación contextual de variables irrelevantes del estudio, lo que implicó la modificación de algunos de los elementos perceptibles dentro de cada tarea, introduciendo los siguientes dualismos y efectos:

- Tarea 1: tras la disolución de un soluto granular (colorante en polvo), se realiza la de un soluto continuo (colorante líquido).

- Tarea 2: tras la mezcla del alcohol y del agua, se realiza la del alcohol y agua coloreados.

- Tarea 3: tras la experimentación del aire (gas incoloro), se «juega» con un gas coloreado, el dióxido de nitrógeno, introducido en una jeringa.

\section{ANÁLISIS DE LOS DATOS}

\section{Tratamiento cualitativo de datos: identificación y descripción de las categorías empíricas}

A partir de los ítems del cuestionario, se construyeron 14 variables. En la tabla 1 se muestran los nombres de las variables en la primera columna y su descripción en la segunda. 
Tabla 1.

Cuestionario y nombre de las variables generadas con descripción de su contenido

\begin{tabular}{|c|c|c|}
\hline Variable & & Contenido \\
\hline AGA & \multicolumn{2}{|c|}{$\begin{array}{l}\text { Tras mostrar una paleta de acuarela, un pincel y un vasito con agua, se moja el pincel en agua y se comprueba } \\
\text { que ahora sí pinta. (Preguntas: ¿Qué hace el agua? ¿Cómo lo hace?) }\end{array}$} \\
\hline AMA & \multirow[b]{2}{*}{$\underset{S}{S}$} & $\begin{array}{l}\text { Se muestra un vasito de disolución amarilla obtenido al limpiar el pincel con acua- } \\
\text { rela amarilla. (Pregunta: ¿Cómo verías el interior del vasito amarillo si tuvieras un } \\
\text { potente microscopio?) }\end{array}$ \\
\hline AMP & & $\begin{array}{l}\text { Se echa una pequeña gota de agua amarilla en un vaso de agua clara sin que tenga } \\
\text { lugar cambio apreciable de color. (Pregunta: ¿Dónde está la gota de color amarillo } \\
\text { si se ve todo transparente? ¿Cómo verías el interior de este vasito si tuvieras un potente } \\
\text { microscopio?) }\end{array}$ \\
\hline VER & \multicolumn{2}{|c|}{$\begin{array}{l}\text { Se echa una gota de colorante líquido en un vaso con } 50 \mathrm{ml} \text { aproximadamente de agua a temperatura ambiente } \\
\text { y otra gota en otro vaso con la misma cantidad de agua caliente. (Pregunta: ¿Por qué se difumina en el agua } \\
\text { caliente más rápidamente el color verde?) }\end{array}$} \\
\hline TEM & \multicolumn{2}{|c|}{ Pregunta: ¿Cómo verías el interior de estos vasitos con un microscopio? } \\
\hline ALA & $\Rightarrow$ & $\begin{array}{l}\text { Se muestra un tubo de ensayo de aproximadamente } 80 \mathrm{~cm} \text { de altura y } 1,5 \mathrm{~cm} \text { de } \\
\text { diámetro, un bote de agua destilada y otro de alcohol. Se agrega agua hasta más o } \\
\text { menos la mitad de este y a continuación se agrega despacio el alcohol inclinando } \\
\text { ligeramente el tubo para evitar que se mezclen. El estudiante marca el nivel final } \\
\text { de líquido mediante un rotulador y se cierra el tubo con un tapón de corcho. (Pre- } \\
\text { gunta: ¿Cambiará la altura del tubo cuando lo agitemos?) }\end{array}$ \\
\hline PAL & \multicolumn{2}{|c|}{$\begin{array}{l}\text { Se agita el tubo y se constata la disminución de la altura total. (Pregunta: ¿Habrá cambiado también el peso } \\
\text { total del tubo?) }\end{array}$} \\
\hline ALP & \multicolumn{2}{|c|}{ Pregunta: Dibuja lo que verías en el interior del tubo si tuvieras un potente microscopio antes y después de agitarlo. } \\
\hline ACA & \multicolumn{2}{|c|}{$\begin{array}{l}\text { Se repite el proceso detallado para la variable ALA pero utilizando ahora agua y alcohol coloreados (con distin- } \\
\text { to color). (Pregunta: ¿Crees que al agitar ahora ocurrirá lo mismo que cuando eran transparentes?) }\end{array}$} \\
\hline ACP & \multicolumn{2}{|c|}{$\begin{array}{l}\text { Se agita el tubo y se constata la disminución de la altura total. (Pregunta: Dibuja lo que verías en el interior de } \\
\text { tubo si tuvieras un potente microscopio antes y después de agitar el agua y el alcohol coloreados) }\end{array}$} \\
\hline PAI & \multicolumn{2}{|c|}{$\begin{array}{l}\text { Se pesa un globo vacío y se solicitan las previsiones sobre el nuevo peso cuando se infle. (Pregunta: ¿Cambiará } \\
\text { el peso del globo si lo inflamos?) }\end{array}$} \\
\hline AAN & \multirow{2}{*}{ 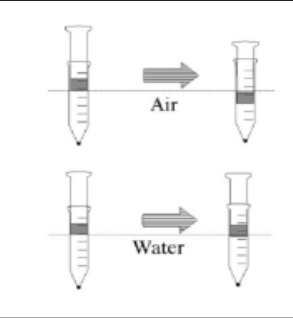 } & $\begin{array}{l}\text { Se aprieta el globo con las manos mostrando la disminución del volumen ocupa- } \\
\text { do. Se hace también el símil con una jeringa con aire tapada por su extremo y se } \\
\text { comprueba que } 5 \mathrm{ml} \text { son reducidos fácilmente a } 1 \mathrm{ml} \text {. (Pregunta: Dibuja lo que } \\
\text { verías en el interior de la jeringa si tuvieras un microscopio muy potente antes y después } \\
\text { de empujar el émbolo) }\end{array}$ \\
\hline $\mathrm{APO}$ & & $\begin{array}{l}\text { Se llena otra jeringa con agua hasta la misma cantidad y se muestra su incompre- } \\
\text { sibilidad. (Pregunta: Dibuja cómo verías el aire y el agua si tuvieras un microscopio } \\
\text { muy potente intentando explicar las distintas compresibilidades) }\end{array}$ \\
\hline GCO & \multicolumn{2}{|c|}{$\begin{array}{l}\text { Se introduce cobre y ácido nítrico en un matraz quitasatos conectado a una jeringa hasta obtener gas rojizo } \\
\text { de dióxido de nitrógeno. Se tapa rápidamente el matraz con un tapón de corcho. Cuando todo el matraz esté } \\
\text { saturado de gas, se succiona con la jeringa. Se muestra la compresibilidad del gas rojizo. (Pregunta: Dibuja } \\
\text { cómo verías el gas rojizo en el interior de la jeringa si tuvieras un microscopio muy potente antes y después de empujar } \\
\text { el émbolo) }\end{array}$} \\
\hline
\end{tabular}

En cada variable se identificaron las respuestas de los estudiantes, se agruparon en categorías mediante procesos inductivos de semejanzas y diferencias entre ellas y posteriormente estas categorías se 
ordenaron jerárquicamente desde las más simples a las más complejas, dando lugar a la construcción de un módulo de categorías empiricas para cada una de las variables identificadas en el cuestionario.

En el establecimiento del orden jerárquico se utilizó como principal criterio la intuición del investigador para detectar en las respuestas elaboraciones que progresivamente se alejan de los propios datos percibidos en las tareas. Otros criterios se basaron en:

- Restarle importancia a los términos o significantes a costa de los significados atribuidos a estos.

- Diferenciar dentro de cada grupo de respuestas semejantes, aportadas por los alumnos después de la contraprueba, a los sujetos que mostraban respuestas previas diferentes.

A modo de ejemplo, en la tabla 2, se muestra la construcción de la variable AMA, a partir de los ítems 1.5 y 1.6 del cuestionario, donde se solicitaba al estudiante un dibujo y una explicación de cómo imagina el interior de un vasito de color amarillo. Vemos que esta variable tiene 4 niveles o categorías posibles. La categoría 1 se corresponde con dibujos dominados por los aspectos perceptivos (dibujos continuos o continuos con manchas) mientras que la categoría 4 recoge aquellos dibujos donde tanto el agua como la acuarela se representan con puntitos. Los estudiantes ubicados en cada categoría se identifican con un número (E1...E31) seguido de la letra A o B, según provenga de su respuesta antes o después de la intervención didáctica. Así, el estudiante 1 (E1) adquiere el valor 3 en esta variable AMA tanto antes como después de la intervención didáctica (véase que E1A y E1B están situados en el modelo 6-categoría 3 de la variable).

Tabla 2.

Construcción de la variable AMA

\begin{tabular}{|c|c|c|}
\hline \multicolumn{3}{|c|}{ Módulo 3 Imagen de la disolución de un sólido granular } \\
\hline & & \\
\hline & & \\
\hline \multicolumn{3}{|c|}{$\begin{array}{l}\text { 3.1 Dominados por la percepción. Hacen dibujos que se corresponden enteramente con esta. } \\
\text { Modelo 1: Continuo amarillo-manchas: Dibujo donde aparecen zonas amarillentas con distinta intensidad, que incluso } \\
\text { pueden llegar a ser transparentes. Representa el agua más o menos coloreada, según la intensidad de la zona. E5A, E7A, } \\
\text { E8A, E11A, E12A, E13A, E15A, E17A, E26A, E27A, E28A, E31A. } \\
\text { Modelo 2: Continuo amarillo: Dibujo donde el amarillo se distribuye uniformemente por toda la gota. E16A, E23A. }\end{array}$} \\
\hline \multicolumn{3}{|c|}{$\begin{array}{l}\text { 3.2 Aún dominados por la percepción. Introducen elementos que no se corresponden enteramente con ella. } \\
\text { Modelo 3: Continuo amarillo-puntos amarillos de acuarela: Dibujo donde, sobre un fondo amarillo que representa el } \\
\text { agua coloreada, destacan puntitos que simbolizan los «trocitos de acuarela sin llegar a disolverse»E14A, E19A, E22A, } \\
\text { E7B, E8B, E11B, E13B, E15B, E16B, E17B, E19B, E22B, E23B, E31B. } \\
\text { Modelo 4: Continuo amarillo-puntos amarillos de agua y acuarela: Dibujo donde, sobre un fondo amarillo que represen- } \\
\text { ta el agua coloreada, destacan puntitos que simbolizan «partículas de acuarela». E25A. }\end{array}$} \\
\hline \multicolumn{3}{|c|}{$\begin{array}{l}\text { 3.3 Transforman los datos perceptivos, lo que les lleva a hacer modelos con fondos transparentes. } \\
\text { Modelo 5: Continuo transparente-puntos amarillos de agua y acuarela: Dibujo donde, sobre un fondo transparente que } \\
\text { representa al agua, destacan puntitos amarillos que representan las "partículas del agua que han agarrado la acuarela». } \\
\text { E9A, E24A, E30A, E12P, E18P. } \\
\text { Modelo 6: Continuo transparente-puntos diversos de «cosas que pueden estar en el agua»: Dibujo donde, sobre un fondo } \\
\text { transparente que representa al agua, destacan puntos de distintas formas que representan «las partículas de todo lo que } \\
\text { puede estar en el agua» (oxígeno, hidrógeno, microorganismos, cloro, partículas de acuarela). E1A, E2A, E3A, E4A, } \\
\text { E10A, E18A, E20A, E29A, E1B, E2B, E3B, E5B, E24B, E25B, E26B. } \\
\text { Modelo 7: Continuo transparente-puntos amarillos de acuarela: Dibujo donde, sobre un fondo transparente que repre- } \\
\text { senta al agua, destacan puntos amarillos que representan «las partículas de acuarela». E6A, E21A, E4B, E6B, E9B, } \\
\text { E10B, E14B, E21B, E27B, E28B, E30B. }\end{array}$} \\
\hline
\end{tabular}


Módulo 3 Imagen de la disolución de un sólido granular

\begin{tabular}{|c|c|c|}
\hline Items & Variable & Contenido \\
\hline $1,5-1,6$ & AMA & Sólido granular/Dibujo y explicación \\
\hline
\end{tabular}

3.4. Transforman los datos perceptivos, lo que les lleva a hacer dibujos donde hay también transformación para el fondo transparente de agua.

Modelo 8: Puntos para el agua - puntos para la acuarela: Dibujo donde, sobre un fondo indeterminado, aparecen puntos que representan las partículas del agua y otros puntos que representan las de acuarela. En los huecos se puede concebir que no hay huecos. E20B, E29B.

Tras cada categoría se muestran los estudiantes ubicados en ella. Se identifica a los estudiantes con un número (E1...E31) seguido de la letra A o B, según sea su categoría antes o después de la intervención didáctica.

De este modo, cada estudiante quedó identificado por un número en cada una de las variables, alcanzando una matriz de datos E_PRE a partir de las respuestas previas a la intervención educativa y otra matriz de datos E_POS con las respuestas posteriores a esta. Para distinguir las variables pertenecientes a sendas matrices, les ańadimos las letras A o B. Así, AMA_A y AMA_B se refieren a la misma variable AMA recogida antes y después de la intervención didáctica respectivamente.

Tanto la matriz de datos E_PRE como la E_POS constan de 31 sujetos x 16 variables. Las 16 variables proceden de las 14 identificadas en el cuestionario más las variables personales SEXO y RT (rendimiento total del cuestionario). La variable RT es simplemente la suma aritmética de los valores de las 14 variables construidas del análisis de los cuestionarios.

Cabe aclarar que las 14 variables del cuestionario son variables categoriales ordinales y que el número de categorías es distinto en cada variable. El valor con el que cada estudiante queda representado en cada variable no indica más que una relación de orden con la categoría representada. El análisis cuantitativo posterior permite dilucidar problemas relacionados con el grado de coherencia del proceso de categorización seguido.

\section{Tratamiento cuantitativo de datos}

Para llegar a identificar los niveles explicativos de los estudiantes se realizó un estudio exploratorio multivariable mediante el programa estadístico SPSS Statistics 20.0 por ser uno de los programas más potentes y disponer de un gran número de módulos para el tratamiento multivariable de datos experimentales. El estudio comprendió los siguientes apartados, que se aplicaron tanto para la matriz E_PRE como a la E_POS:

- Análisis de correlaciones bivariadas entre variables.

- Análisis factorial de variables.

- Agrupamiento de sujetos por sus comportamientos semejantes. Análisis clúster.

- Análisis de correspondencias múltiples e identificación de los niveles explicativos de los estudiantes.

Nos referiremos únicamente a los dos últimos, por ser los más significativos para los propósitos de este estudio.

Agrupamiento de sujetos por sus comportamientos semejantes: análisis clúster. El análisis de clúster es una herramienta exploratoria diseñada para mostrar grupos (o clústeres) de estudiantes, dentro de un conjunto de datos que, de otra manera, no podrían ser visibles. El módulo del SPSS «Análisis de conglomerado en dos fases» da la opción de elegir el número de clúster que se desea construir, o bien calcula por defecto el mejor número de agrupaciones, que será el que presente un criterio bayesiano de 
Schwarz (BIC) inferior. En nuestro caso, tanto para la matriz E_PRE como para la E_POS, el programa elige tres clústeres. En la tabla 3 se muestra el conglomerado en el que queda mejor ubicado cada estudiante.

Tabla 3.

Pertenencia a los conglomerados

de los estudiantes $(\mathrm{N}=31)$ antes y después de la intervención didáctica

\begin{tabular}{|c|c|c|c|c|c|}
\hline Estudiante & $\begin{array}{l}\text { Clúster } \\
\text { (E_PRE) }\end{array}$ & $\begin{array}{c}\text { Clúster } \\
\text { (E_POS) }\end{array}$ & Estudiante & $\begin{array}{c}\text { Clúster } \\
\left(E_{-} P R E\right)\end{array}$ & $\begin{array}{c}\text { Clúster } \\
\text { (E_POS) }\end{array}$ \\
\hline 1 & 2 & 2 & 16 & 1 & 1 \\
\hline 2 & 2 & 2 & 17 & 1 & 1 \\
\hline 3 & 2 & 2 & 18 & 2 & 2 \\
\hline 4 & 2 & 2 & 19 & 1 & 1 \\
\hline 5 & 3 & 1 & 20 & 2 & 3 \\
\hline 6 & 3 & 2 & 21 & 3 & 3 \\
\hline 7 & 1 & 1 & 22 & 3 & 1 \\
\hline 8 & 1 & 1 & 23 & 1 & 1 \\
\hline 9 & 3 & 2 & 24 & 2 & 2 \\
\hline 10 & 3 & 1 & 25 & 1 & 2 \\
\hline 11 & 2 & 1 & 26 & 1 & 1 \\
\hline 12 & 2 & 1 & 27 & 1 & 2 \\
\hline 13 & 2 & 1 & 28 & 1 & 2 \\
\hline 14 & 2 & 2 & 29 & 2 & 3 \\
\hline 15 & 2 & 1 & 30 & 3 & 2 \\
\hline & & & 31 & 2 & 1 \\
\hline
\end{tabular}

Hay que aclarar que los valores obtenidos solo sirven para comparar la ubicación de cada estudiante en el grupo en cada momento en que se administró el cuestionario. El hecho de que la correlación entre ambas variables no sea máxima indica las múltiples direcciones en que se produce el aprendizaje. Por ejemplo, los estudiantes mejores antes de la intervención didáctica no tienen por qué seguir siendo los mejores tras esta. De hecho, aproximadamente la mitad de los estudiantes cambian sus posiciones relativas respecto al grupo tras la intervención didáctica.

Análisis de correspondencias múltiples e identificación de los niveles explicativos de los estudiantes. Como se señaló con anterioridad, las 14 variables categóricas construidas a partir del cuestionario son variables categoriales ordinales, construidas intuitivamente, con las limitaciones que presentan a la hora de establecer cualquier comparación entre sus valores o categorías. Por tanto, interesó indagar:

- Cómo se relacionan los distintos valores o categorías de dichas variables.

- Qué variables están bien construidas o, por el contrario, cuáles tienen inversiones o lagunas en los órdenes categoriales.

- Cómo modificar los módulos categoriales para que sean verdaderamente representativos de la evolución cognitiva de los sujetos y no de los esquemas de partida de la entrevistadora.

La técnica de análisis de datos adecuada para estos objetivos es el análisis de correspondencias múltiples (ACM). Sus consideraciones son de carácter geométrico, está dentro de las técnicas descriptivas de la estadística y permite el paso de lo cualitativo a lo cuantitativo, de lo heterogéneo a una construcción de orden estructural (Cornejo, 1988, citado en Benarroch, 1998). 
Para realizar el ACM con nuestras variables se utilizó el módulo «Escalamiento óptimo» del SPSS, introduciendo como variables de análisis únicamente las más significativas del análisis de variables realizado previamente. El ACM ofrece como resumen un estudio de la fiabilidad del análisis a través de la estimación del coeficiente del alfa de Cronbach.

Dado que el alfa de Cronbach promedio proporciona información sobre las relaciones entre elementos individuales de la escala, al comparar su valor para E_PRE (alfa $=0,742)$ y E_POS (alfa $=$ $0,864)$, se confirma de nuevo la mayor coherencia e interrelación entre las modalidades tras la intervención didáctica. Considerando que en el ámbito educativo suele ser aceptable un coeficiente de fiabilidad de 0,6 o superior, los valores de alfa obtenidos indican además la alta fiabilidad del cuestionario tanto antes como después de la intervención didáctica.

El programa presenta la opción de proporcionar un gráfico en el que se proyectan todas las categorías de respuestas de las variables seleccionadas. Mediante este gráfico se procedió a un análisis de las variables categoriales ordinales, en cuanto a la distribución de sus categorías, su capacidad de discriminación y sus posibles inversiones. Por razones de espacio se omite dicho análisis en este trabajo.

El programa permite otro gráfico donde los puntos de objetos (estudiantes) se proyectan sobre el espacio definido por ambas dimensiones del ACM a partir de las variables más significativas y homogéneas. La ubicación de los estudiantes en este espacio gráfico es muy importante para nuestro estudio, dado que este ha sido construido por la correspondencia entre las categorías de las variables más determinantes y homogéneas de la matriz de datos. Tanto es así, que adjudicándole a cada estudiante el valor definido por su posición en este espacio gráfico, se obtienen los niveles explicativos antes y después de la intervención didáctica (variables NC_PRE y NC_POS respectivamente).

En este trabajo solo se muestra el gráfico con la proyección de los estudiantes tras la intervención didáctica (figura 2), a partir del cual, por intervalización de ambos ejes, se alcanzaron los valores de la variable NC_POS. Estos valores se muestran en la tabla 4, junto a los valores de la variable NC_PRE.

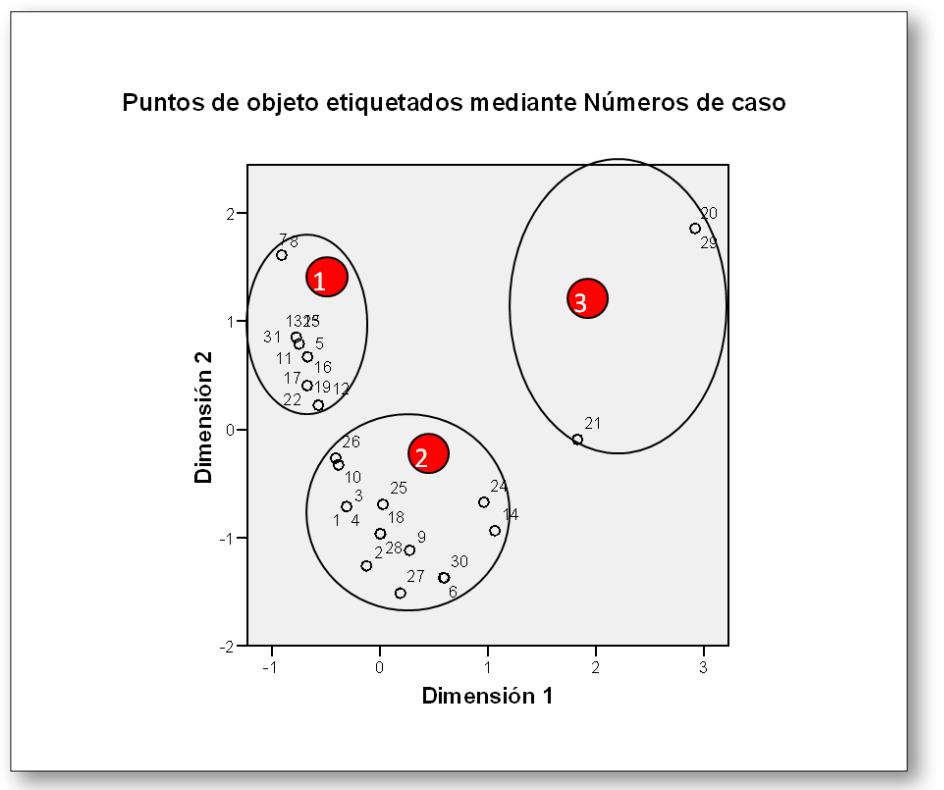

Fig. 2. Análisis de correspondencias múltiples (E_POS). Ubicación de los estudiantes en el espacio definido por las variables más significativas (AGA_B, PIN_B, AMA_B, AMP_B, VER_B, TEM_B, ALP_B). 
Tabla 4 .

Nivel explicativo de los estudiantes antes y después de la intervención didáctica

\begin{tabular}{|c|c|c|}
\hline \multirow{2}{*}{ Estudiante } & NC_PRE & NC_POS \\
\hline 1 & 2 & 2 \\
\hline 2 & 2 & 2 \\
\hline 3 & 2 & 2 \\
\hline 4 & 1 & 2 \\
\hline 5 & 3 & 1 \\
\hline 6 & 3 & 2 \\
\hline 7 & 1 & 1 \\
\hline 8 & 1 & 1 \\
\hline 9 & 3 & 2 \\
\hline 10 & 3 & 2 \\
\hline 11 & 2 & 1 \\
\hline 12 & 2 & 1 \\
\hline 13 & 2 & 1 \\
\hline 14 & 2 & 2 \\
\hline 15 & 2 & 1 \\
\hline
\end{tabular}

\begin{tabular}{|c|c|c|}
\hline Estudiante & NC_PRE & NC_POS \\
\hline 16 & 1 & 1 \\
\hline 17 & 1 & 1 \\
\hline 18 & 2 & 2 \\
\hline 19 & 1 & 1 \\
\hline 20 & 2 & 3 \\
\hline 21 & 3 & 3 \\
\hline 22 & 3 & 1 \\
\hline 23 & 1 & 1 \\
\hline 24 & 1 & 2 \\
\hline 25 & 1 & 2 \\
\hline 26 & 1 & 2 \\
\hline 27 & 2 & 2 \\
\hline 28 & 1 & 2 \\
\hline 29 & 2 & 3 \\
\hline 30 & 3 & 2 \\
\hline 31 & 2 & 1 \\
\hline
\end{tabular}

Hay que destacar la gran coincidencia de los valores de NC_PRE y NC_POS (tabla 4) con los de CLUSTER_A y CLUSTER_B (tabla 3) respectivamente, que se manifiestan en correlaciones de 0,901 y 0,910 altamente significativas. De hecho, solo los estudiantes E4, E24 y E27 tienen valores distintos entre CLUSTER_A y NC_PRE, y solo los estudiantes E10 y E26 difieren en sus valores de CLUSTER_B y NC_POS. Evidentemente, esta coincidencia está manifestando tanto la bondad de los datos como la de los análisis realizados.

\section{RESULTADOS}

En este trabajo el objetivo es evaluar el aprendizaje de conocimientos específicos y el aprendizaje de competencias científicas. Para el primero se utiliza la variable RT (rendimiento total del cuestionario) y para el segundo la variable NC (nivel explicativo o competencial del estudiante). La primera ha sido construida mediante la suma aritmética de los valores de todas las variables, sin discriminar entre ellas, mientras que la segunda ha sido construida a partir únicamente de las variables más significativas, que son aquellas en las que los estudiantes tienen un comportamiento estable y homogéneo.

\section{Aprendizaje realizado según RT (rendimiento total en el cuestionario)}

Aquí se consideran:

- RT_PRE: rendimiento acumulado en el cuestionario antes de la intervención didáctica. Es la suma aritmética de los valores de todas las variables categoriales ordinales de la matriz de datos E_PRE.

- RT_POS: rendimiento acumulado en el cuestionario después de la intervención didáctica. Es la suma aritmética de los valores de todas las variables categoriales ordinales de la matriz de datos E_POS. 
- GANANCIA_RT: aprendizaje experimentado durante la intervención didáctica referido a la totalidad de las variables. Se calcula como la diferencia entre RT_POS y RT_PRE.

Realizados estos cálculos, se encuentra que la variable GANANCIA_RT oscila entre 12 y 35 puntos, siendo su media 20,81, lo que indica que el grupo de estudiantes ha experimentado un aprendizaje neto positivo, corroborado por que la diferencia de medias entre RT_PRE y RT_POS es significativa al 99\% de confianza (prueba T de medias entre muestras relacionadas $=23,471$; grado de significación $=0,000$ ).

Se puede concluir que la diferencia de medias entre los rendimientos totales en el cuestionario antes y después de la intervención didáctica es significativa, y que por lo tanto ha habido aprendizaje de contenidos específicos. No obstante, este es ligeramente inferior a medida que el nivel inicial es superior, como se comprueba al comparar los valores de la $\mathrm{T}$ de Student entre grupos de estudiantes que pertenecen a distintos niveles.

\section{Aprendizaje realizado según NC (nivel explicativo-competencial del estudiante)}

Para poder comparar los niveles explicativos alcanzados por los estudiantes antes y después de la intervención didáctica (variables NC_PRE y NC_POS), se debe previamente redimensionar en un nuevo espacio bidimensional construido mediante un ACM de componentes principales que incluya todas las variables anteriores y posteriores conjuntamente.

Si en el espacio gráfico del nuevo ACM se proyectan las variables que representan los niveles explicativos de los estudiantes antes y después de la intervención didáctica (NC_PRE y NC_POS), se obtiene la figura 3, sobre la cual se recodifican dichas variables, tal y como se recoge en la parte inferior de esta figura.

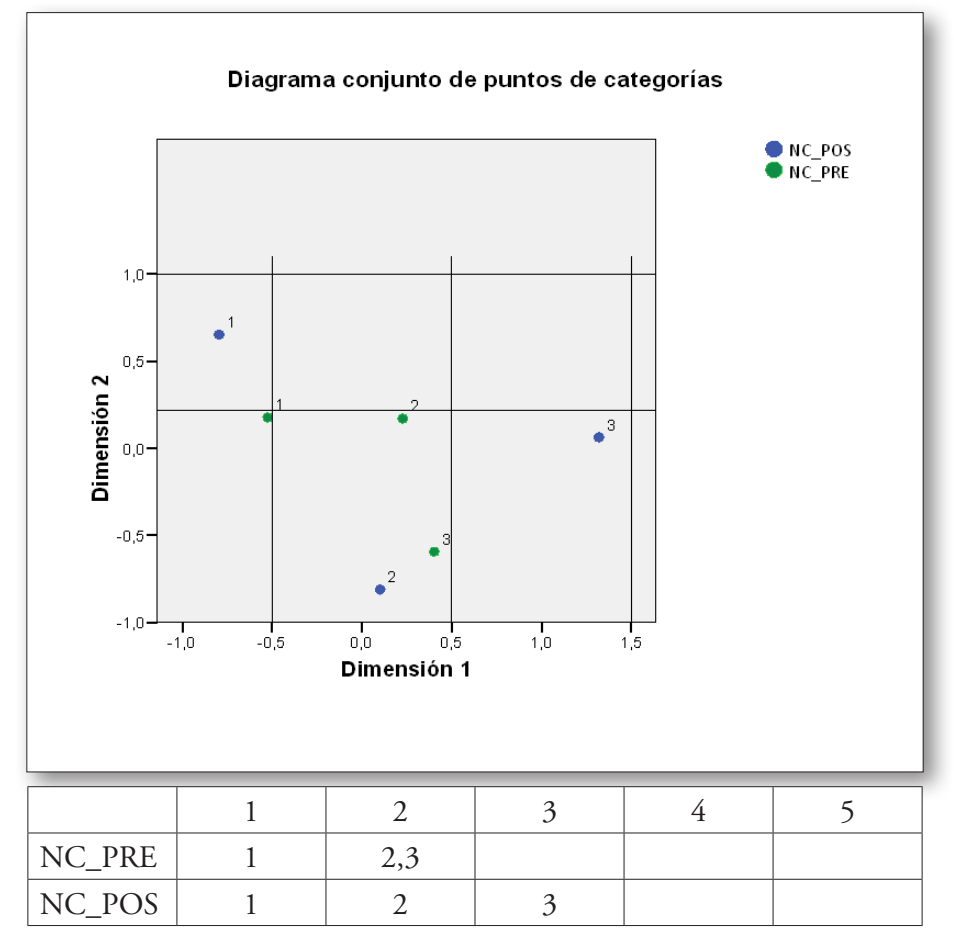

Fig. 3. Análisis de correspondencias múltiples (E_PRE_POS). Ubicación de las categorías de las variables NC_POS y NC_PRE en el espacio definido por las variables más significativas (AMA_A, AMA_B, AMP_B, VER_B y ALP_B). 
Sustituyendo los valores iniciales de estas variables por los sugeridos por la ubicación en el espacio gráfico de estos, se alcanzan nuevas variables denominadas «nivel previo transformado» (NC_PRE_T) $\mathrm{y}$ «nivel posterior transformado» (NC_POS_T), cuyos valores se muestran en la tabla 5.

Tabla 5.

Valores de las variables NC_PRE_T y NC_POS_T

\begin{tabular}{|c|c|c|c|c|c|}
\hline Estudiante & $N C \_P R E \_T$ & NC_POS_T & Estudiante & $N C \_P R E \_T$ & $N C \_P O S \_T$ \\
\hline 1 & 2 & 2 & 16 & 1 & 1 \\
\hline 2 & 2 & 2 & 17 & 1 & 1 \\
\hline 3 & 2 & 2 & 18 & 2 & 2 \\
\hline 4 & 1 & 2 & 19 & 1 & 1 \\
\hline 5 & 2 & 1 & 20 & 2 & 3 \\
\hline 6 & 2 & 2 & 21 & 2 & 3 \\
\hline 7 & 1 & 1 & 22 & 2 & 1 \\
\hline 8 & 1 & 1 & 23 & 1 & 1 \\
\hline 9 & 2 & 2 & 24 & 1 & 2 \\
\hline 10 & 2 & 2 & 25 & 1 & 2 \\
\hline 11 & 2 & 1 & 26 & 1 & 2 \\
\hline 12 & 2 & 1 & 27 & 2 & 2 \\
\hline 13 & 2 & 1 & 28 & 1 & 2 \\
\hline 14 & 2 & 2 & 29 & 2 & 3 \\
\hline 15 & 2 & 1 & 30 & 2 & 2 \\
\hline & & & 31 & 2 & 1 \\
\hline
\end{tabular}

A la vista de estas nuevas variables, se puede concluir que:

a) Ningún estudiante adopta un nivel superior a 2 antes de la intervención didáctica.

b) Solo tres estudiantes adoptan el nivel 3 tras la intervención didáctica.

c) Ningún estudiante supera el nivel explicativo 3, ni siquiera después de la intervención didáctica.

La tabla 6 de contingencia entre NC_PRE_T y NC_POS_T describe los progresos en términos de niveles explicativos.

Tabla 6.

Tabla de contingencia NC_PRE_T ${ }^{*}$ NC_POS_T

\begin{tabular}{|l|l|r|r|r|c|}
\hline \multicolumn{2}{|c|}{} & \multicolumn{3}{c|}{ NC_POS_T } & \multirow{2}{*}{ Total } \\
\cline { 3 - 6 } \multicolumn{2}{|c|}{} & 1 & 2 & 3 & \\
\hline NC_PRE_T & 1 & 6 & 5 & 0 & 11 \\
\hline & 2 & 7 & 10 & 3 & 20 \\
\hline Total & 13 & 15 & 3 & 31 \\
\hline
\end{tabular}

Como puede verse en la tabla 6, hay 11 alumnos que inicialmente tienen un nivel previo 1 , cuyos niveles finales pueden ser: 
- Nivel 1 (estudiantes 7, 8, 16, 17, 19 y 23). Total: 6 .

- Nivel 2 (estudiantes 4, 24, 25, 26 y 28). Total: 5.

Y hay 20 casos que tienen un nivel de entrada 2, cuyos niveles finales pueden ser:

- Nivel 1 (estudiante 5, 11, 12, 13, 15, 22 y 31). Total: 7.

- Nivel 2 (estudiantes 1, 2, 3, 6, 9, 10, 14, 18, 27 y 30). Total: 10.

- Nivel 3 (estudiantes 20, 21 y 29). Total: 3.

En consecuencia, 7 alumnos experimentan un retroceso en su nivel explicativo-competencial, 16 permanecen en el que estaban y solo 8 de 31 (el 26\% de los estudiantes) experimentan un progreso gracias a la intervención didáctica. Los valores medios de NC_PRE_T y NC_POS_T son respectivamente 1,65 y 1,68, lo que indica la limitación del progreso global. Este progreso no los lleva en ningún caso a superar el nivel explicativo 3 en la construcción del conocimiento sobre la naturaleza corpuscular de la materia.

\section{DISCUSIÓN DE RESULTADOS Y CONCLUSIONES}

Los análisis realizados en este trabajo ponen de manifiesto dos resultados relevantes:

a) Que los valores del «rendimiento total del cuestionario» (variable RT) tras la intervención didáctica son significativamente superiores a los obtenidos con anterioridad a esta.

b) Y que, por el contrario, los valores en el «nivel explicativo-competencial» (variable NC_T) tras la intervención didáctica no distan mucho de los obtenidos con anterioridad a esta.

La cuestión que suscita estos resultados es ¿a qué se debe esta diferencia? Para responder a esta cuestión hemos de acudir a la propia naturaleza de las variables citadas. Por un lado, recordamos que la variable RT es simplemente la suma aritmética de todos los valores alcanzados por cada estudiante en las variables del cuestionario. En su construcción, no se realiza ningún tipo de discriminación entre variables significativas o no significativas y, por tanto, no está restringida a aquellas que representan un comportamiento repetido, generalizado y adaptado a las contrapruebas y variaciones contextuales relevantes e irrelevantes introducidas en las tareas. Por el contrario, si se comparan las variables que más pesan en su cálculo, se podría concluir que la ganancia de esta variable RT es consecuencia principal de la adquisición de la información memorística que el estudiante ha podido acumular mediante la intervención educativa.

En la figura 3, se muestra que las variables más significativas del estudio (esto es, aquellas en las que el comportamiento relativo de cada estudiante respecto a su grupo es más uniforme) son: AMA_A, AMA_B, AMP_B, VER_B y ALP_B. Del total de las 28 variables extraídas de los cuestionarios (14 para el cuestionario antes y 14 para el cuestionario posterior), solo 5 son las más significativas. En el cálculo de la ganancia competencial o del avance de niveles explicativos, solo se tienen en cuenta estas variables más significativas. En cambio, en el cálculo de los RT, se han tenido en cuenta todas las variables, las 5 significativas y las 23 no significativas, y por ello se afirma que la mejora en los rendimientos totales se debe principalmente a la adquisición de la información memorística.

Por el contrario, la variable NC_T ha sido construida utilizando únicamente las variables que muestran un comportamiento coherente, homogéneo y estable del estudiante ante las estrategias de variación con que fue diseñado el cuestionario. Cabe destacar que su número es pequeño, lo que podría tener la lectura de que para alcanzar dicho comportamiento coherente se requiere un coste elevado de enseñanza. 
De acuerdo con el marco teórico utilizado en el trabajo, se podría suponer que la ganancia de RT es una medida posible del aprendizaje de contenidos específicos, mientras que la ganancia de la variable NC_T es una medida posible del aprendizaje de las competencias del estudiante ante tareas que pueden ser explicadas en términos corpusculares.

Admitido esto, el valor de este trabajo estriba principalmente en haber sido capaces de diseñar una metodología para discriminar entre aprendizaje de contenidos específicos y aprendizaje de competencias. Para ello ha sido necesario aplicar un conjunto exigente de estrategias entre las que destacamos las siguientes:

1. Diseño de un cuestionario, usando para ello estrategias de confrontación y de variación contextual.

2. Aplicación del cuestionario antes y después de la intervención didáctica.

3. Análisis de las respuestas de los estudiantes, agrupación de estas y jerarquización, alcanzando a construir las variables categoriales ordinales asociadas al cuestionario.

4. Análisis cuantitativo de datos, importante sobre todo para identificar las variables más significativas que se deben introducir en un análisis de correspondencias múltiples a través del cual se consiga determinar los niveles explicativos de los estudiantes.

5. Tras la aplicación de los dos apartados anteriores a los resultados de los cuestionarios antes y después de la intervención didáctica, se habrían alcanzado los valores de los niveles explicativos tanto anteriores como posteriores a esta, pero estos deben ser recodificados mediante un nuevo análisis de correspondencias múltiples que parta de la consideración de todas las variables ante y post. Así se alcanzan los niveles explicativos transformados (o proyectados sobre el espacio gráfico de todas las variables anteriores y posteriores).

6. Por último, análisis de la ganancia en los niveles explicativos transformados y de la ganancia en el resultado acumulado del cuestionario, para valorar el aprendizaje de competencias y de contenidos respectivamente.

Ante la inevitable pregunta acerca de por qué el aprendizaje de competencias es más dificultoso que el de contenidos específicos, en relación con el comportamiento del estudiante ante tareas que pueden ser explicadas en términos corpusculares, son posibles muchas respuestas dependiendo del modelo cognoscitivo que se utilice. Por ejemplo, para Costamagna (2005) los estudiantes simplemente no usan activamente gran parte de lo que saben; para Galavosky y Adúriz Bravo (2001) esto es debido a la interferencia del pensamiento cotidiano en la activación del conocimiento científico. Nosotros pensamos que la actuación competente depende de esquemas de conocimiento generales y específicos. Si bien estos últimos podrían depender de la enseñanza del propio contenido, los esquemas de conocimiento generales tienen un carácter más transversal y exigen periodos de tiempo amplios que permitan la adquisición de los procedimientos cognitivos necesarios para usar los conceptos específicos de forma flexible, controlada y adecuada a los contextos prácticos. Por ello, afirmamos que enseñar competencias requiere una enseńanza costosa, que implica mucho tiempo, además de profesorado experto, capaz de diseñar tareas en contextos específicos similares a aquellos donde esperamos que se demuestre la competencia deseada. Además, en relación con la competencia de «explicar fenómenos que pueden ser explicados en términos corpusculares», es posible que los estudiantes de 12 y 13 años tengan dificultades específicas relacionadas con la necesidad de alcanzar el nivel tercero de la evolución cognoscitiva, en el que se exige el esquema asimilador formado por "partículas y huecos entre estas». Para Benarroch (2001), este esquema de la discontinuidad no es fácil de alcanzar, debido en parte a las exigencias cognitivas que conlleva. En definitiva, dificultades tanto específicas como generales podrían estar en la base de estos resultados, aparentemente buenos, si en ellos se incluye la información memorizada por los estudiantes. 


\section{REFERENCIAS BIBLIOGRÁFICAS}

Benarroch, A. (1998). Las explicaciones de los estudiantes sobre las manifestaciones corpusculares de la materia. Tesis Doctoral. Universidad de Granada. Disponible en línea: <http://hdl.handle. net/10481/14909> (consulta: 06/10/2014).

Benarroch, A. (2000a). Del modelo cinético-corpuscular a los modelos atómicos. Reflexiones didácticas. Alambique. Didáctica de las Ciencias Experimentales, 23, pp. 95-108.

Benarroch, A. (2000b). El desarrollo cognoscitivo de los estudiantes en el área de la naturaleza corpuscular de la materia. Enseñanza de las ciencias, 18(2), pp. 235-247.

Benarroch, A. (2001). Una interpretación del desarrollo cognoscitivo de los alumnos en el área de la naturaleza corpuscular de la materia. Enseñanza de las Ciencias, 19(1), pp. 123-135.

Costamagna, A. (2005). El valor de la metaevaluación del cambio conceptual: una experiencia didáctica. Enseñanza de las ciencias, 23(3), pp. 419-430.

Galagovsky, I. y A. Adúriz Bravo (2001). Modelos y analogías en la enseñanza de las ciencias naturales. El concepto de modelo didáctico analógico. Enseñanza de las Ciencias, 19 (2), pp. 231-242.

Jiménez Gómez, E., A. Benarroch y N. Marín (2006). Evaluation of the degree of coherence found in students. Conceptions concerning the particulate nature of matter. Journal of Research in Science Teaching, 43(6), pp. 577-598.

http://dx.doi.org/10.1002/tea.20130

Marín, N. (1994). Elementos cognoscitivos dependientes del contenido. Revista Interuniversitaria de Formación del Profesorado, 20, pp. 195-208.

Marín, N., A. Benarroch y E. Jiménez Gómez (2000). What is the relationship between Social Constructivism and Piagetian Constructivism? An analysis of the characteristics of the ideas within both theories. International Journal of Science Education, 22 (3), pp. 225-238.

http://dx.doi.org/10.1080/095006900289840

OCDE (2010). Pisa 2009. Programa para la Evaluación Internacional de los Alumnos. Informe Espańol.

Piaget, J. y B. Inhelder (1982). El desarrollo de las cantidades en el niño. Barcelona: Hogar del Libro (versión original: Le Developpement Des Quantités Physiques Chez L'enfant. Delachaux \& Niestlé, Lausanne, Suiza, 1941).

Pro, A. y J. Rodríguez (2010). Aprender competencias en una propuesta para la enseñanza de los circuitos eléctricos en educación primaria. Enseñanza de las Ciencias, 28(3), pp. 385-404.

Тово́n, S. (2006). Formación basada en competencias: Pensamiento complejo, diseño curricular y didáctica. Bogotá: Ecoe.

Zabala, A. y L. Arnau (2007). 11 ideas clave. Cómo aprender y enseñar competencias. Barcelona: Editorial Graó. 


\title{
Learning of scientific competencies vs learning of scientific content. An assessment proposal
}

\author{
Alicia Benarroch \\ Universidad de Granada \\ aliciabb@ugr.es \\ Graciela Núñez \\ Universidad Nacional de San Juan. Argentina \\ gnunez@ffha.unsj.edu.ar
}

New 21 st century curriculum trends focus on skills training and assessment, be they general cognitive, emotional, social, instrumental, etc. This is an ambitious goal in response to current socioeconomic demands, which mostly call for decision-making capacity, autonomy, flexibility in the face of changing demands and teamwork, just as skills and knowledge were required during the industrial revolution to handle the machinery.

In this paper, we focus on learning of cognitive scientific skills and a possible methodology for its assessment, distinguishing it from learning of specific contents, both in relation to the Corpuscular Model of Matter (MCM).

In the theoretical framework, we turned to research on students' preconceptions to find a construct to serve as a correlate of the concept of competence and especially its evaluation. This construct was the explanatory schema, which, like the skill concept, is manifested with three observables: repetition, generalisation and differentiation. Skills learning, thus, involves changing the level of explanatory schemas. So, we postulated that:

The new topic of scientific competencies could be associated with the concept of explanatory schemata, previously used in research on preconceptions of students.

Considering the above, the assessment of scientific skills learning will be based on the pre-post change in the explanatory levels achieved by students before and after educational intervention. This is equivalent to stating that skills learning involves mobilising explanatory levels.

Assessing the learning of specific contents could be carried out on the basis of the total performance achieved in the questionnaire applied, without distinguishing between the responses that came from the explanatory schemata and those that did not.

In this work, the sample consists of a group $(\mathrm{N}=31)$ of pupils 12-13 years old from San Juan province (Argentina), who underwent a teaching process of 30 hours duration on MCM with one of the authors in a natural classroom context.

Before and after the educational intervention, the Benarroch (1998) questionnaire was administered to delimit i) explanatory skills levels (NC) and ii) overall performance (RT) of each of the pupils.

Based on the survey items, we constructed 14 ordinal categorical variables, to which the personal variables SEX and RT were added. The RT variable is simply the arithmetic sum of the values of the 14 variables constructed from the analysis of the questionnaires. Thus, we obtained two data matrices E_PRE and E_POS, both consisting of 31 subjects x 16 variables. Both jointly underwent a multivariate exploratory study using the SPSS Statistics 20.0 program, which allowed us to distinguish the significant variables, which met the conditions of repetition, generalisation and differentiation, from those that did not. These significant variables were then used to assess the gain in the explanatory-competence level, while in the assessments of improvements in overall performance we used all the variables, without distinguishing significant from non-significant.

Two pertinent results were obtained:

- The values for «Total Questionnaire Performance» after the educational action are significantly higher than those obtained beforehand.

- In contrast, the values for «Explanatory Skills Level» after didactic intervention were not far removed from those previously obtained thereof.

These results highlight the difficulty of learning skills compared to the considerably less difficult learning of specific content.

We conclude that skills learning requires an expensive education, which takes a long time, as well as expert teaching staff, able to design tasks in specific contexts progressively similar to those where we expect the desired skill to be demonstrated. Moreover, in relation to the skill of «explaining phenomena that can be described in corpuscular terms», it is possible that 12-13-year-old pupils may have specific difficulties related with the need to reach the third level of cognitive development, which calls for the assimilator schema consisting of "particles and gaps between them». For us, this discontinuity schema is not easy to achieve, partly due to the cognitive demands involved. In short, both specific and general difficulties could be the basis of these apparently good results, if one takes into account the information memorised by the pupils. 